Quelle: Sebastian Lux, SpringerMedizin.de, basierend auf: Virtueller Workshop: Akne und Rosazea, 27. Fortbildungswoche für praktische Dermatologie und Venerologie (FOBI Digital), 07.-

11.07.2020, digitaler Kongress

Rosazea-Subtypen

\title{
Symptomorientierte Lösungen bei Rosazea
}

Da viele Rosazea-Subtypen parallel auftreten, findet die Klassifizierung anhand der klinischen Symptome zunehmend Anerkennung. Für die Behandlung der verschiedenen Ausprägungen sei der 2016 publizierte Therapiealgorithmus [1] sehr sinnvoll, so der Tübinger Prof. Dr. Martin Schaller. In seinem Vortrag fokussierte er auf besondere Herausforderungen.

Für die okuläre Rosazea mangelt es an zugelassenen Therapien. Schaller hat daher zusammen mit Tübinger Kollegen aus der Ophthalmologie eine kleine Pilotstudie mit zehn Patienten mit leichter bis mittelschwerer Rosazea am Auge durchgeführt, die unter anderem Blepharitis, Chalazien und Teleangiektasien der Augenlider hatten [2].

\section{Off-Label-Behandlung}

Alle Patienten sollten einmal täglich eine etwa halberbsengroße Menge einer $1 \%$ igen Ivermectin-Creme auf das geschlossene Lid einmassieren keinesfalls ins Auge, so Schaller. Das Follow-up betrug durchschnittlich acht Monate. Schon nach 16 Wochen Behandlung besserten sich konjunktivale Rötung, Blepharitis sowie Beschaffenheit und Ausdrückbarkeit des Meibum-Sekrets signifikant. Schaller wies darauf hin, dass man für diese Off-Label-Behandlung mit den Patienten unbedingt den Beipackzettel besprechen müsse, der eine Anwendung am Auge nicht vorsieht.

Sowohl bei Flushings als auch bei persistierenden Erythemen empfahl
Schaller den Betablocker Carvedilol in Standarddosis. Für besonders schwere inflammatorische Läsionen gebe es mit dem Makrolid Azithromycin eine "Therapie der letzten Wahl“, erklärte Schaller.

\section{Rosacea fulminans}

Eine schwere Verlaufsform ist die Rosacea fulminans. „Sie ist selten, aber mittlerweile überblicke ich 20 bis 30 Patienten“, erklärte Schaller. Fast alle Betroffenen seien Frauen. Gute Therapieerfolge werden mit Azithromycin erzielt, das sich auch in der Schwangerschaft eignet - oder bei Frauen, die (noch) kein Isotretinoin einnehmen können oder wollen. Erstaunlich sei dabei das schnelle Ansprechen, so Schaller.

》) Für die okuläre Rosazea mangelt es an zugelassenen Therapien

Bei Morbus Morbihan, einer Sonderform der Rosazea mit ödematösen zentrofazialen Veränderungen, ist Isotretinoin $10 \mathrm{mg}$ plus Antihistaminikum über ein bis zwei Jahre Schallers präferierte Therapieoption. „Damit können
Sie Patienten mit dieser entstellenden Erkrankung sehr gut helfen", sagte Schaller. Wichtig sei, auf die lange Therapiedauer hinzuweisen, da nur $\mathrm{Pa}$ tienten, die die Behandlung über ein Jahr durchhalten, eine signifikante Verbesserung erreichen.

\section{Literatur}

1. Schaller M et al (2016) Rosacea Management: Update on general measures and topical treatment options. J Dtsch Dermato Ges 14(S6):17-28, https://doi.org/10.1111/ ddg. 13143

2. Sobolewska B et al (2020) Efficacy of Topical Ivermectin for the Treatment of Cutaneous and Ocular Rosacea.Ocular Immunology and Inflammation. https://doi.org/10.1080/0927 3948.2020.1727531

Hinweis des Verlags. Der Verlag bleibt in Hinblick auf geografische Zuordnungen und Gebietsbezeichnungen in veröffentlichten Karten und Institutsadressen neutral.

hautnah $2020 \cdot 19: 134$

https://doi.org/10.1007/s12326-020 00409-3

(c) Springer-Verlag GmbH Austria, ein Teil von Springer Nature 2020

Hier steht eine Anzeige. 